\title{
Erratum to: Clinician's guide to prevention and treatment of osteoporosis
}

\author{
F. Cosman • S. J. de Beur • M. S. LeBoff • E. M. Lewiecki • \\ B. Tanner $\cdot$ S. Randall $\cdot$ R. Lindsay
}

Published online: 19 May 2015

(C) International Osteoporosis Foundation and National Osteoporosis Foundation 2015

\section{Erratum to: Osteoporos Int \\ DOI 10.1007/s00198-014-2794-2}

The publisher apologizes for typesetting errors which made the Executive Summary of the Clinician's Guide to Prevention and Treatment of Osteoporosis more difficult to read. A corrected version of this section of the Guide is given here.

The online version of the original article can be found at http://dx.doi.org/ 10.1007/s00198-014-2794-2.

F. Cosman $(\bowtie) \cdot$ R. Lindsay

Helen Hayes Hospital, West Haverstraw, NY, USA

e-mail: cosmanf@helenhayeshosp.org

\section{S. J. de Beur}

Johns Hopkins Bayview Medical Center, Baltimore, MD, USA

M. S. LeBoff

Brigham and Women's Hospital, Boston, MA, USA

E. M. Lewiecki

New Mexico Clinical Research and Osteoporosis Center,

Albuquerque, NM, USA

B. Tanner

Vanderbilt University Medical Center, Nashville, TN, USA

S. Randall

National Osteoporosis Foundation, Washington, DC, USA

F. Cosman $\cdot$ R. Lindsay

Department of Medicine, Columbia University, New York, NY, USA

\section{Executive summary}

Osteoporosis is a silent disease until it is complicated by fractures - fractures that occur following minimal trauma or, in some cases, with no trauma. Fractures are common and place an enormous medical and personal burden on the aging individuals who suffer them and take a major economic toll on the nation. Osteoporosis can be prevented, diagnosed and treated before fractures occur. Importantly, even after the first fracture has occurred, there are effective treatments to decrease the risk of further fractures. Prevention, detection and treatment of osteoporosis should be a mandate of primary care providers.

Since NOF first published the Guide in 1999, it has become increasingly clear that many patients are not being given appropriate information about prevention and many patients are not receiving appropriate testing to diagnose osteoporosis or establish osteoporosis risk. Most importantly, many patients who have osteoporosis-related fractures are not being diagnosed with osteoporosis and are not receiving any of the FDA-approved, effective therapies.

This Guide offers concise recommendations regarding prevention, risk assessment, diagnosis and treatment of osteoporosis in postmenopausal women and men age 50 and older. It includes indications for bone densitometry and fracture risk thresholds for intervention with pharmacologic agents. The absolute risk thresholds at which consideration of osteoporosis treatment is recommended were guided by a cost-effectiveness analysis. 
Synopsis of major recommendations to the clinician :

Recommendations apply to postmenopausal women and men age 50 and older.

\section{Universal recommendations}

- Counsel on the risk of osteoporosis and related fractures.

- Advise on a diet that includes adequate amounts of total calcium intake (1000 mg per day for men 50 $70 ; 1200 \mathrm{mg}$ per day for women 51 and older and men 71 and older), incorporating dietary supplements if diet is insufficient.

- Advise on vitamin D intake (800-1000 IU per day), including supplements if necessary for individuals age 50 and older.

- Recommend regular weight-bearing and musclestrengthening exercise to improve agility, strength, posture and balance; maintain or improve bone strength; and reduce the risk of falls and fractures.

- Assess risk factors for falls and offer appropriate modifications (e.g. home safety assessment, balance training exercises, correction of vitamin D insufficiency, avoidance of central nervous system depressant medications, careful monitoring of anti-hypertensive medication and visual correction when needed).

- Advise on cessation of tobacco smoking and avoidance of excessive alcohol intake.

\section{Diagnostic assessment}

- Measure height annually, preferably with a wall mounted stadiometer.

- BMD testing should be performed:

-In women age 65 and older and men age 70 and older.

-In postmenopausal women and men above age 50-69, based on risk factor profile.

-In postmenopausal women and men age 50 and older who have had an adult age fracture, to diagnose and determine degree of osteoporosis.

-At dual x-ray absorptiometry (DXA) facilities using accepted quality assurance measures.

- Vertebral imaging should be performed:

-In all women age 70 and older and all men age 80 and older if T-score is $\leq-1.0$ at the lumbar spine, total hip or femoral neck.

-In women age 65 to 69 and men age 70 to 79 if T-score is $\leq-1.5$ at the lumbar spine, total hip or femoral neck.
-In postmenopausal women and men age 50 and older with specific risk factors:

- Low trauma fracture during adulthood (age 50 and older)

- Historical height loss of 1.5 in. $(4 \mathrm{~cm})$ or more Defined as the difference between the current height and peak height at age 20

- Prospective height loss of 0.8 in. $(2 \mathrm{~cm})$ or more Defined as the difference between the current height and a previously documented height measurement

- Recent or ongoing long term glucocorticoid treatment

-If bone density testing is not available, vertebral imaging may be considered based on age alone.

- Check for secondary causes of osteoporosis.

- Biochemical markers of bone turnover can aid in risk assessment and serve as an additional monitoring tool when treatment is initiated.

\section{Monitoring patients}

- Perform BMD testing 1 to 2 years after initiating medical therapy for osteoporosis and every 2 years thereafter.

-More frequent BMD testing may be warranted in certain clinical situations.

-The interval between repeat BMD screenings may be longer for patients without major risk factors and who have an initial T-score in the normal or upper low bone mass range.

- Biochemical markers can be repeated to determine if treatment is producing expected effect.

\section{Pharmacologic treatment recommendations}

- Initiate pharmacologic treatment:

-In those with hip or vertebral (clinical or asymptomatic) fractures.

- In those with T-scores $\leq-2.5$ at the femoral neck, total hip or lumbar spine by DXA.

-In postmenopausal women and men age 50 and older with low bone mass ( $\mathrm{T}$-score between -1.0 and -2.5 , osteopenia) at the femoral neck, total hip or lumbar spine by DXA and a 10-year hip fracture probability $\geq 3 \%$ or a 10 -year major osteoporosis-related fracture probability $\geq 20 \%$ based on the U.S.-adapted WHO absolute fracture risk model (FRAX ${ }^{\circledR}$; www.NOF.org and www. shef.ac.uk/FRAX). 
- Current FDA-approved pharmacologic options for osteoporosis are bisphosphonates (alendronate, ibandronate, risedronate and zoledronic acid), calcitonin, estrogen agonist/antagonist (raloxifene), estrogens and/or hormone therapy, tissue-selective estrogen complex (conjugated estrogens/bazedoxifene), parathyroid hormone 1-34 (teriparatide) and RANK ligand inhibitor (denosumab).

- No pharmacologic therapy should be considered indefinite in duration. After the initial treatment period, which depends on the pharmacologic agent, a comprehensive risk assessment should be performed. There is no uniform recommendation that applies to all patients and duration decisions need to be individualized.

- In adults age 50 and older, after a fracture, institute appropriate risk assessment and treatment measures for osteoporosis as indicated. Fracture liaison service (FLS) programs, where patients with recent fractures may be referred for care coordination and transition management, have demonstrated improvement in the quality of care delivered. 